\title{
THE EFFECT OF NUMERICAL PARAMETERS ON EDDIES IN OCEANIC OVERFLOWS: A LABORATORY AND NUMERICAL STUDY
}

\author{
SHANON M. RECKINGER, THOMAS H. GIBSON, FRED M. HOHMAN, THERESA J. MORRISON, \\ SCOTT J. RECKINGER \& MATEUS CARVALHO \\ Fairfield University, United States of America.
}

\begin{abstract}
Overflows in the ocean occur when dense water flows down a continental slope into less dense ambient water. It is important to study idealized and small-scale models, which allow for confidence and control of parameters. The work presented here is a direct qualitative and quantitative comparison between physical laboratory experiments and lab-scale numerical simulations. Physical parameters are varied, including the Coriolis parameter, the inflow density, and the inflow volumetric flow rate. Laboratory experiments are conducted using a rotating square tank and high-resolution camera mounted on the table in the rotating reference frame. Video results are digitized in order to compare directly to numerical simulations. The MIT General Circulation Model (MITgcm), a three-dimensional ocean model, is used for the direct numerical simulations corresponding to the specific laboratory experiments. It was found that the MITgcm was not a good match to laboratory experiments when physical parameters fell within the high eddy activity regime. However, a more extensive resolution study is needed to understand this fully. The MITgcm simulations did provide a good qualitative and quantitative match to laboratory experiments run in a low eddy activity regime. In all cases, the MITgcm simulations had more eddy activity than the laboratory experiments.
\end{abstract}

Keywords: density-driven currents, lab-scale, numerical parameters, ocean modelling, overflows.

\section{INTRODUCTION}

Oceanic overflows are density driven currents that flow down topographical features and mix with the surrounding ocean. Dense water forms for a variety of reasons including cooling, evaporation, and salt addition (due to brine rejection during ice formation). After the dense water is produced, which typically occurs in a semi-enclosed region, it can flow down narrow straits or continental slopes until it eventually reaches the bottom of the ocean or enters the open ocean when it reaches a neutral buoyancy level [1]. Examples of oceanic overflows include the Nordic deep overflows such as in the Denmark Strait [2, 3] and Faroe Bank Channel [4], Antarctic deep overflows such as from the Weddell [5] and Ross seas [6, 7], and the intermediate subtropical overflows such as from the Red [8-10], and Mediterranean seas [11].

The upcoming Intergovernmental Panel on Climate Change (IPCC) highest resolution global ocean simulations use a $0.1^{\circ}$ horizontal resolution, with grid spacing ranging from 5-15 km, depending on the location. Overflow channels can be as small as $5 \mathrm{~km}$ wide (e.g. Red Sea Overflow). Therefore, most overflows are not resolved, and none of the overflows' mixing and entrainment processes are resolved because these scales are on the order of meters [12]. Additionally, most global ocean circulation models solve the hydrostatic primitive equations, so vertical mixing must be parameterized. Thus, a good understanding of the processes regulating overflows is crucial, given that they are still a challenge in ocean modeling.

\section{BACKGROUND}

The dense waters associated with overflows affect the properties of intermediate and deep water and significantly influence large-scale ocean circulation [13]. There have been 
numerous studies that have looked at the dynamics of overflows in realistic domains under real-world forcing. Some studies compare the effects of various overflow parameters on ocean general circulation models (OGCMs) with particular focus on thermohaline circulation [14]. Others compare different OGCMs in their accuracy at matching observations in the overflow regions [15]. Some studies focus on regional models, where high resolution simulations are run in a realistic domain to understand how resolution and numerical parameters affect the dynamics of the overflows [16]. These studies are essential for improving representations of overflows in OGCMs. However, comparison to observational data is limited. There have been some extensive observational studies, such as the work of [4], but many overflow regions do not have adequate observational data available for comparison against models.

Laboratory scale experimental overflow studies provide a controlled environment for understanding the dynamics of density driven currents. Cenedese et al. [17] ran a large set of overflow experiments in a rotating tank over a sloping bottom. They varied many physical parameters such as inflow density, inflow velocity, rotation rate of the tank, and angle of the sloping bottom. They compared laboratory results to a theoretical, steady state model and classified the different overflows based on their physical properties into three regimes: laminar flow, waves, and eddies. Etling [18] also separates overflows into different regimes (vortex and plume) by using a large (13 m diameter) tank at the Coriolis laboratory in Grenoble, France. A handful of studies directly compare their experimental results with a numerical model. Sommeria and Decamp [19] quantitatively compared some initial results using the Grenoble turntable to MICOM (an isopycnal model) where Hallberg's mixing parameterization was used [20]. They found that the main features of the gravity currents were well represented by the numerical model, but the velocities were too high and the plume thickness was too small. Davies et al. [21] also directly compared laboratory experiments with a numerical model. They were able to characterize the flow by the formation of two counteracting eddies and a bottom boundary current, which qualitatively matched the numerical model results, but quantitative agreement was weak. The numerical model was a three dimensional model using generalized sigma coordinate and a Galerkin finite element method [22]. Wobus et al. [23] also performed a direct comparison of laboratory and numerical model results. Their laboratory setup was quite different from the other experiments, with a conical geometry. They used POLCOMS, a three-dimensional model with sigma coordinates for comparison. Their qualitative comparison was quite extensive and resulted in several important conclusions. First, a no slip boundary condition was a much better match to the laboratory results than the model using quadratic bottom drag. Second, running the code in the nonhydrostatic mode was not necessary for capturing the cascading plume. Lastly, the laboratory and numerical results generally showed excellent agreement.

The MITgcm is used in this study for lab-scale simulations due to its ability to simulate small scales using its non-hydrostatic formulation [24]. Previous work using the MITgcm to simulate lab-scale experiments include simulating a differentially heated rotating annulus experiment [25]. Their experimental setup, particularly the rotating turntable, is identical to the setup used for the study presented in this paper. In another study, the MITgcm was used to simulate two lab-scale test cases: (a) lock release gravity currents in a flat bottom tank, and (b) the propagation and breaking of internal solitary waves in a tank with a sloping bottom [26]. The MITgcm had good qualitative agreement with the laboratory results in these studies. The non-hydrostatic formulation in the MITgem was used in Ref. [27] for high-resolution simulations of an idealized overflow at real scales; however, it was not a direct numerical simulation or at lab-scale. 


\section{METHODS}

\subsection{Laboratory methods}

Overflows depend on many physical characteristics. For this model, three parameters are varied: the Coriolis parameter, the inflow density, and the inflow volumetric flow rate. The parameters are adjusted such that each case has a unique combination of values. Three rotation speeds, two inflow rates, and three different inflow densities are used in different combinations for eighteen total cases. For each given case, an experiment is performed three times to ensure accurate results and reproducibility.

The three rotation speeds are $0,5,10 \mathrm{rpm}$. The three fluid inflow densities are 1.024, 1.035, and $1.043 \mathrm{~kg} / \mathrm{m}^{3}$. The two inflow rates are 0.50 and $1.10 \mathrm{~kg} / \mathrm{m}^{3}$. A summary of the cases is shown in Table 1. A visual summary of the overflow plumes for a subset of the cases is shown in Fig. 2 with the appropriate case numbers labeled.

The laboratory setup is comprised of a square acrylic tank (approximately $40 \times 40 \times 6 \mathrm{~cm}^{3}$ ), which sits on a rotating turntable designed and built by Dana Sigall [28]. A square tank is used to avoid optical distortion for side views of fluid motion in the tank. The drive mechanism is a friction wheel driven by a variable speed motor. The rotation rate is measured using a tachometer. Slip rings allow for auxiliary equipment to be powered via the turntable. A variable-angle, sloping-bottom acrylic insert sits on the bottom of the tank. It is painted white to ensure the video camera only records the dyed dense water on the top of the slope and none of the water that leaks over the edges of the slope and mixes beneath the slope. A water pump (with a variable flow rate) is set on the rotating platform to inject dense fluid into the tank. In the top right corner sits an embayment, which is a small rectangular box ( $4 \mathrm{~cm}$ deep with a base that is $5 \mathrm{~cm}$ by $5 \mathrm{~cm}$ ) where dense water is pumped into and spills out onto the sloping bottom (see Fig. 1). Along with the tank, on top of the table there is also a smaller reservoir holding the dense water. The tank is filled with freshwater, and is then spun up to reach a solid-body rotation (for 30 minutes) before the dense water is pumped into the embayment from the reservoir. Dense fluid is injected into the embayment by manually switching on the pump. The density of the inflow is controlled by salinity differences. The dense inflow water then spills over the opening onto a uniform slope, which is at an angle.

Table 1: A summary of all case parameters.

\begin{tabular}{llllllll}
\hline & $\begin{array}{l}\text { Pump flow } \\
\text { Case }\end{array}$ & rate, $\mathbf{~ c m}^{\mathbf{3} / \mathbf{s}}$ & $\begin{array}{l}\text { Rotation } \\
\text { rate, } \mathbf{r p m}\end{array}$ & $\begin{array}{l}\text { Inflow } \\
\text { Density, } \mathbf{g} / \mathbf{c m}^{\mathbf{3}}\end{array}$ & $\begin{array}{l}\text { Pump flow } \\
\text { Case }\end{array}$ & $\begin{array}{l}\text { rate, } \mathbf{c m}^{\mathbf{3} / \mathbf{s}} \\
\text { Rotation } \\
\text { rate, rpm }\end{array}$ & $\begin{array}{l}\text { Inflow } \\
\text { Density, } \\
\mathbf{g} / \mathbf{c m}^{\mathbf{3}}\end{array}$ \\
\hline 1 & 0.5 & 10 & 1.024 & 3 & 0.5 & 10 & 1.035 \\
4 & 0.5 & 10 & 1.043 & 5 & 1.1 & 10 & 1.024 \\
7 & 1.1 & 10 & 1.053 & 8 & 1.1 & 10 & 1.043 \\
9 & 0.5 & 5 & 1.024 & 11 & 0.5 & 5 & 1.035 \\
12 & 0.5 & 5 & 1.043 & 13 & 1.1 & 5 & 1.024 \\
15 & 1.1 & 5 & 1.035 & 16 & 1.1 & 5 & 1.043 \\
17 & 0.5 & 0 & 1.024 & 19 & 0.5 & 0 & 1.035 \\
20 & 0.5 & 0 & 1.045 & 21 & 1.1 & 0 & 1.024 \\
23 & 1.1 & 0 & 1.035 & 24 & 1.1 & 0 & 1.043 \\
\hline
\end{tabular}


The dense plume either reaches its neutral buoyancy level and hits the west wall or it reaches the bottom of the slope.

Video data collection is done with a GoPro Hero 3+ located in the rotating frame of reference above the tank and is turned on remotely via a smart phone app. Video is shot using 1080p high-definition video at 60 frames per second. Data is extracted from the video by discretizing the frames during post processing, allowing direct comparison of various metrics to the numerical results. The dense current is visualized using food dye. The following parameters are varied: rotation rate $\left(f=0-2 \mathrm{~s}^{-1}\right)$, dense flow rate $\left(Q=0.5-1.1 \mathrm{~cm}^{3} / \mathrm{s}\right)$, and inflow density $\left(\mathrm{g}^{\prime}=\mathrm{g}\left(\rho_{2}-\rho_{1}\right) / \rho_{1} \mathrm{~g}=0.135-0.320 \mathrm{~cm} / \mathrm{s}^{2}\right)$. The densities correspond to the ambient fluid density, $\rho_{1}$, and the injected dense fluid density, $\rho_{2}$. This gives the following non-dimensional number ranges: Reynolds, $30<\operatorname{Re}<90$; Rossby, $0.14<$ Ro $<0.86$; and Froude, $0.1<\mathrm{Fr}<0.3$. The Reynolds number is based on the depth of the tank as the characteristic length scale, the viscosity of water, and the post-processed plume speed as the characteristic velocity. The Rossby number is based on the same characteristic length and velocity scales plus the rotation rate, $f$. The Froude number is based on the same characteristic length and velocity scales plus the inflow density, g'. These ranges were chosen to span all three regimes (Laminar, Eddies, and Waves) found in Ref. [17].

\subsection{Numerical methods}

The MIT General Circulation Model (MITgcm) is used for the numerical simulations. The MITgcm's non-hydrostatic formulation allows it to be used for a wide range of scales, including laboratory scales. MITgcm has the flexibility to start at laboratory scales for an idealized system, and to extend the setup to global scales in a realistic domain, which will be the focus of future work. This ocean model is similar to the ocean models used in IPCC simulations. The MITgcm solves the governing equations using a finite volume method with an Arakawa

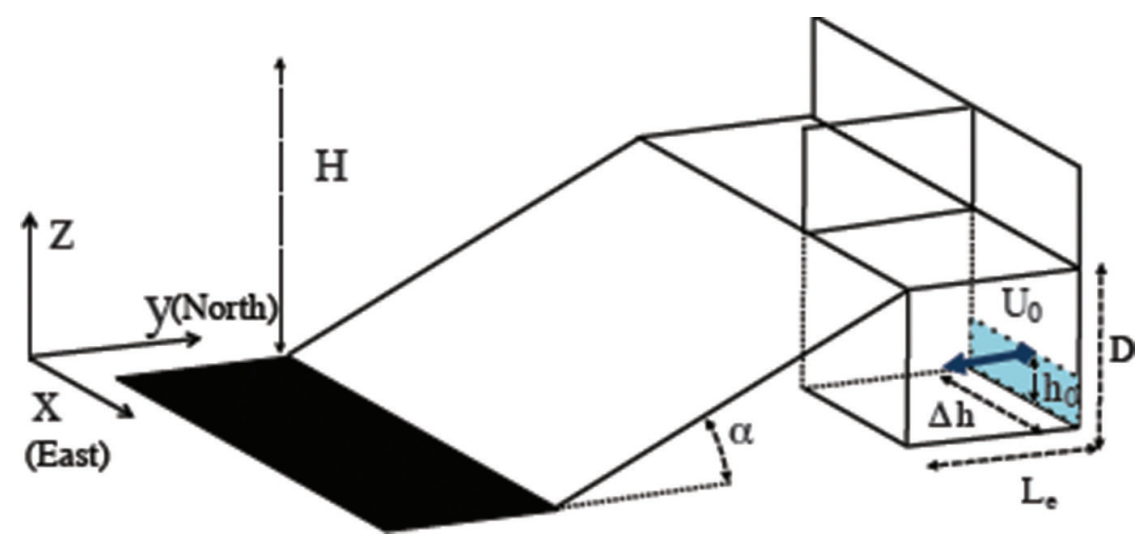

Figure 1: Schematic showing the model domain, with the dense inflow entering in a flatbottomed embayment. The fluid then overflows out the top and down a uniform slope. $H=6 \mathrm{~cm}$ is the depth of the water. $\mathrm{Le}=5 \mathrm{~cm}$ and $\Delta \mathrm{h}=5 \mathrm{~cm}$ are the length and width of the embayment, respectively. $\mathrm{D}=4 \mathrm{~cm}$ is the depth of the embayment. $a=10^{\circ}$ is the angle of the sloping bottom (or $s=\tan \alpha=0.65$ ). $\mathrm{h}_{0}=0.12 \mathrm{~cm}$ is the height of the injected inflow. $U_{0}$ varies based on the inflow rate, $Q$, and inflow area, $\mathrm{A}=h_{0} \Delta h$. 
C-grid discretization scheme. It has been configured to numerically solve the non-hydrostatic incompressible Boussinesq equations for all of the simulations presented here.

The idealized overflow setup, shown in Fig. 1, is based on the Dynamics of Overflow Mixing and Entrainment (DOME) test case [13]. The geometry of the domain, initial conditions, boundary conditions, and system forcing are all the same as in the laboratory experiments. Initial conditions in the simulations have the embayment filled $3 \mathrm{~cm}$ from the bottom with dense fluid to shorten computational time.

In the model domain, numerically prescribed artificial boundaries simulate the rotating tank. No-slip and no-flux boundary conditions are applied at the topography as in Ref. [27], without any additional bottom drag model $(\mathrm{Cd}=0)$, as was done in Ref. [23]. At the northern boundary of the computational domain, specifically at the embayment, a prescribed inflow open boundary condition is applied. In all the simulations, the Orlanski radiative boundary condition is applied at the western open boundary [29]. Along the eastern, southern, and northern shelf boundaries, a no-slip boundary condition is applied.

A resolution study of the sensitivity of the numerical simulations to grid cell size was done. Based on the results of the resolution study, a $200 \times 200 \times 200$ grid is deemed sufficient to fully resolve the plume area for cases with both many and few eddies. This is equivalent to approximately $\Delta \mathrm{x}=2 \mathrm{~mm}$ in the horizontal and $\Delta \mathrm{z}=0.3 \mathrm{~mm}$ in the vertical. Other studies utilizing numerical simulations of laboratory scale overflows have used a wide range of resolutions. Wobus et al. [23] used a resolution of $\Delta \mathrm{x}=5 \mathrm{~mm}$, with a $120 \mathrm{x} 120$ horizontal grid. They had 45 sigma levels, of which 10 were in the frictional boundary layer at the bottom. The smallest layer was $\Delta \mathrm{z}=0.04 \mathrm{~mm}$. Davies et al. [21] used a resolution of $\Delta \mathrm{x}=5.8 \mathrm{~mm}$, with a $129 \times 65$ horizontal grid. They had 11 sigma levels, which meant that the smallest $\Delta \mathrm{z}$ $=3.6 \mathrm{~mm}$ (approximately). The ratio of horizontal to vertical resolutions has not been tested and will be part of future work. Whereas convergence is observed for plume area at a resolution of $200 \times 200 \times 200$, the plume path continues to trend toward the laboratory data up to a resolution of $400 \times 400 \times 400$. Thus, resolution itself is an important numerical parameter for simulating overflow dynamics and will be the focus of future studies.

Work by Reckinger et al. [30], and work cited within, indicates that overflow characteristics drastically depend on numerical parameters like viscosity, diffusivity, and resolution. Even more, these parameters influence one another. In this work, these parameters are set as close to the true laboratory values as possible. The numerical simulations used in this study are all run with molecular viscosity of $v=10^{-6} \mathrm{~m}^{2} / \mathrm{s}$. Wobus et al. [23] used a viscosity twice that of the molecular value and reported that this difference in viscosity was chosen in order to account for impurities in the tap water used in the laboratory. A variety of diffusivities were tested and the one which provided the best match to the laboratory results was $k=1.43 \times 10^{-7}$ $\mathrm{m}^{2} / \mathrm{s}$, which is the thermal diffusivity for water). The molecular diffusivity of salt, $k=1.3 \times$ $10^{-9} \mathrm{~m}^{2} / \mathrm{s}$, produced a plume which had eddies that separated off of the main plume, so it was not used. Since the density difference is due to a salinity difference, the diffusivity value needs to be further explored.

\section{ANALYSIS}

Direct comparisons of laboratory experiments and numerical simulations are done using a variety of metrics. For the laboratory data, the food dye in the video images is used to quantify the spread of the plume over the course of the overflow event. This is done by examining pixel intensity and setting a threshold of $1 \%$ to designate the dense plume. In the equations below this is denoted as $\tau>10^{-2}$, where $\tau(x, y)$ is the vertically-averaged tracer concentration 
as a function of the $x$ and $y$ coordinates. Tracer concentration from the MITgem is vertically summed to mimic the video's top view in the laboratory. This numerical tracer concentration and laboratory pixel intensity is calibrated such that the concentrations or color, respectively, in the embayment matches. The plume area at any moment in time is defined as

$$
P(t)=\frac{\iint_{A} d x d y}{\iint d x d y}
$$

where A is the $x-y$ cross-sectional area where $\tau>10^{-2}$ at a specific time, t. The denominator in the expression above is simply total area of our topographic slope, such that the plume area is the fraction of the domain with inflow water concentration above the threshold.

As was done in Ref. [27] and other similar studies thereafter, we provide the following metric describing the tracer weighted plume path. The tracer-weighted plume path in the $y$-direction at any moment in time is defined as

$$
Y(\mathrm{x})=\frac{\int y \tau(\mathrm{x}, \mathrm{y}) \mathrm{dy}}{\int \tau(\mathrm{x}, \mathrm{y}) \mathrm{dy}}
$$

The slope of the plume path curve has more physical relevance than the global shifts to the east or west. Therefore, when comparing laboratory and numerical cases, the comparison of the slopes is of interest. The plume velocity is defined as $v=d s / d t$, where $s(t)=$ $\left(Y\left(x_{\max }\right)^{2}+x_{\max }^{2}\right)^{1 / 2}$ and $x_{\max }=x_{\max }(t)$ is the maximum horizontal (West-East) plume extent at time, t. In practice, the plume velocity is calculated as the slope of the best fit linear regression line to $s(t)$.

\section{RESULTS}

A summary of a subset of the cases can be found in Fig. 2 with side-by-side images of the plume for laboratory experiments (left) and the numerical simulations (right). Tracer weighted plume path, as shown in eqn (2), is a strong metric for comparison between laboratory experiments and numerical simulations because it is normalized by tracer concentration and, thus, is minimally dependent on how the color matching of the laboratory images is done. When comparing plume paths (Fig. 3), the slope of the plume path curve has more physical
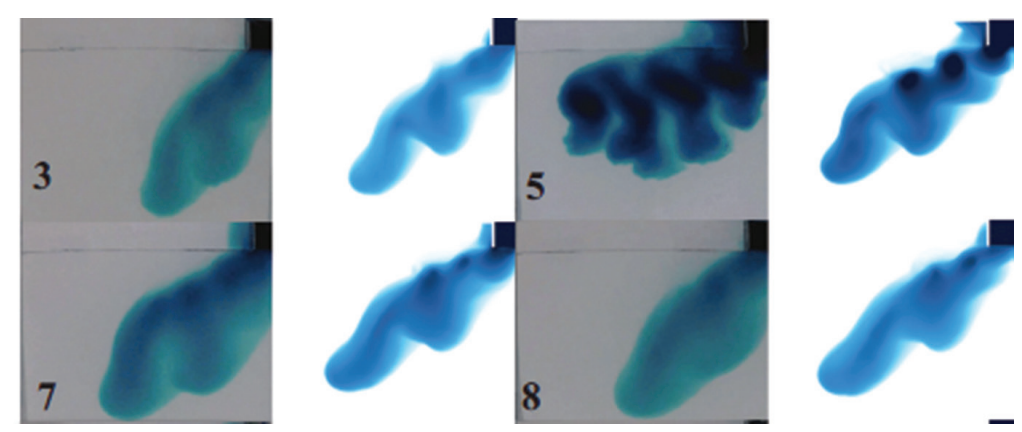

Figure 2: A side-by-side visual comparison of four cases, with the laboratory images (left), the numerical results (right), and the case number indicated. 

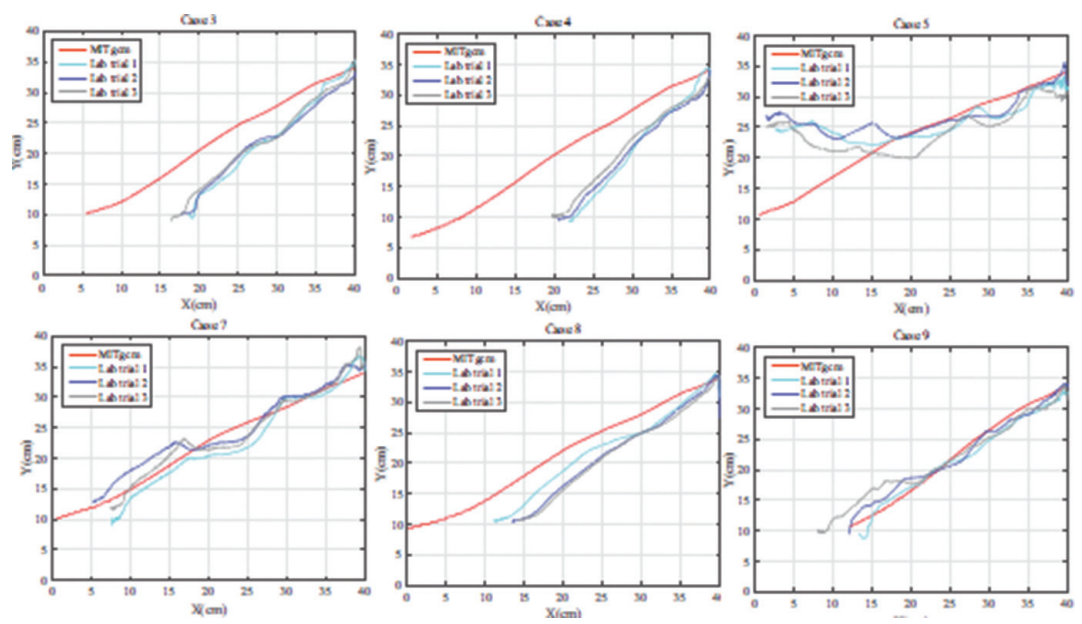

Figure 3: Weighted plume path for selected cases.

relevance than global shifts to the east or west when comparing laboratory and numerical cases. Case 5 (more eddies) and Case 8 (fewer eddies) both show discrepancies in the plume path. For Case 5 (more eddies), the paths are closer at the beginning of the plume's descent, but diverge halfway down the slope. This is a result of the MITgcm having less eddying behavior and mixing than the laboratory experiment. The plume stays denser and less diluted in the MITgcm simulation and so the plume descends further downslope than in the laboratory setup. The same explanation can be given for the other high eddying cases, including Cases 7, 9, and 13. Case 12 also has high eddying behavior, but the laboratory tracer weighted plume path drops below the MITgcm path towards the end of the simulation. For Case 8 (fewer eddies), the laboratory and numerical plume paths closely match, with the laboratory slopes being consistently steeper than in the MITgem simulation. In this case, the MITgem has slightly more mixing, which results in a plume that descends downslope less than in the laboratory. This same result can be found in other cases, including Cases 3, 4, and 11. (Note that the figures do not include multiple cases that show redundant behavior.)

Another consistent difference between laboratory and numerical plume paths is that the MITgcm never captures the initial dip that occurs right when the dense plume leaves the embayment. In many of the cases (e.g. Cases 4, 11, 12, and 15), the plume path slope is steeper as the dense water immediately leaves the embayment (top right of the plots in Fig. 3). This is likely due to the fact that the numerical methods do not consider surface tension effects and do not resolve scales associated with surface tension forces. In the laboratory, surface tension forces at the interface between the dense and ambient fluid at the top of the embayment keep the two from mixing. Once enough dense fluid has been pumped into the embayment such that gravitational effects overcome surface tension, the plume starts descending over the corner of the embayment. The strength of this effect depends on the flow rate and density difference. None of the numerical results capture this because the resolved scales are not small enough and surface tension effects are not considered.

The spread of the plume is measured using plume area, defined in eqn (1) and plotted in Fig. 4. This metric is the most dependent on the color matching algorithm used for post -processing the laboratory images. However, the results do emphasize the differences found for the cases with high and low eddying behavior. For example, Case 5 (more eddies) has a higher 

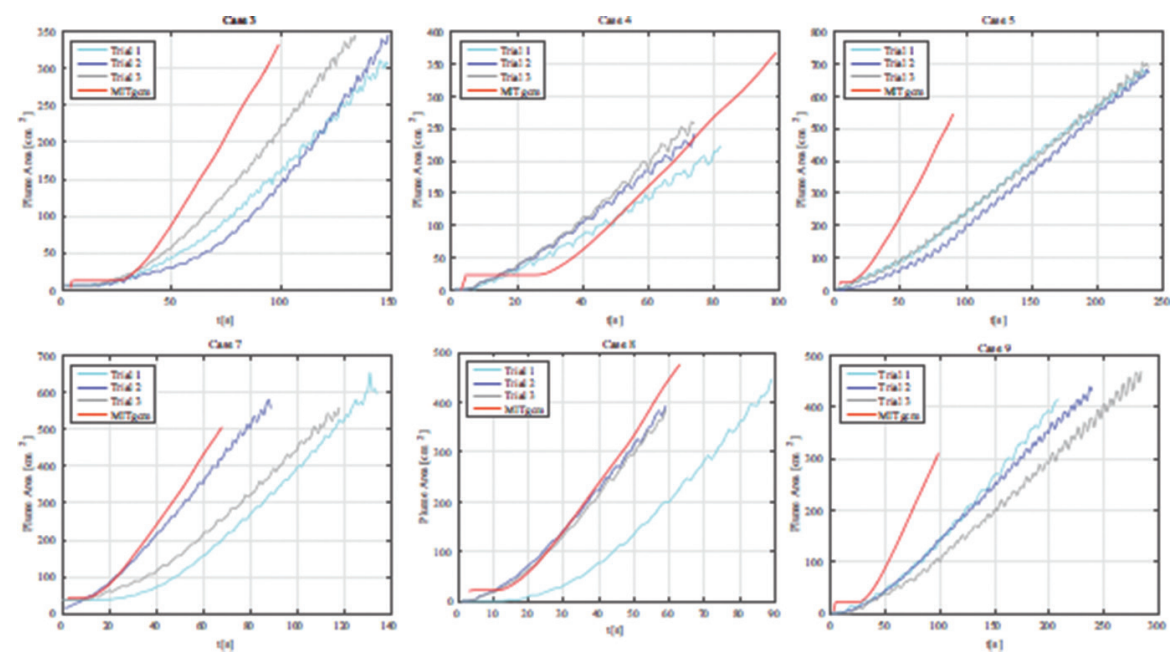

Figure 4: Plume area for selected cases.

discrepancy between laboratory experiments and numerical simulations than Case 8 (fewer eddies). Note that trial 1 in Case 8 appears to be an outlier and should be ignored. The plume area for the cases with high eddy activity (e.g. Cases 5, 7, 9, and 13) all have the same trend, specifically that the laboratory results all spread much less than the numerical simulations, which indicates that the laboratory experiments have much less mixing than the MITgcm. The plume area comparison for Case 12 (high eddying behavior) shows a strong match between laboratory and numerical results. For cases with fewer eddies (e.g. Cases 4, 8, 11, and 15), plume areas generally have similar behavior for laboratory experiments and numerical simulations, particularly in the slope of the plume area curves, representing the growth rate of the plume area (Case 12 behaves similarly to Case 8 in Fig. 4). The non-rotating cases (e.g. Cases 17 and 19) have a particularly poor plume area match between laboratory experiments and numerical simulations. This is a result of a mismatch in the velocity of the plume.

Plume velocity is the final metric used to compare laboratory and numerical results, which is shown in Table 2. The error displayed on each plot is the L $\infty$ error of the MITgcm velocity and the mean velocity from the three laboratory trials, defined as Error $=\mid($ VMIT-Vlab-mean $)$ / Vlab-mean|. Unlike the tracer weighted plume path and plume area, the plume velocity metric shows how the experiments compare temporally. The temporal and spatial scales in the MITgcm simulations were set to be identical to the laboratory experiment. However, plume velocity compares poorly across all cases, with the MITgcm consistently having a higher plume velocity than in the laboratory experiments. The plume velocity metric measures how fast the plume is moving through the ambient fluid in whatever direction the plume path dictates. Thus, it is not a metric that quantifies how fast downslope the plume descends and is not a measure of mixing or entrainment.

The plume velocity further highlights the disparity between the cases with more eddies versus the cases with fewer eddies. The cases with more eddying activity (e.g. Cases 5, 9, 13) all have very high errors when comparing the laboratory and numerical plume velocities. In those cases, the MITgcm plume velocity is 2-3 times bigger than laboratory plume velocity (the errors range between $103.0 \%$ to $171.4 \%$ ). The other two cases that are classified as high eddy activity are Cases 7 and 12, which have relatively low errors in the plume velocity 
Table 2: Comparison of plume velocity from laboratory versus MITgcm for all cases.

\begin{tabular}{lllllll}
\hline Case & $\mathbf{3}$ & $\mathbf{4}$ & $\mathbf{5}$ & $\mathbf{7}$ & $\mathbf{8}$ & $\mathbf{9}$ \\
\hline Lab - Trial 1 (cm/s) & 0.30 & 0.42 & 0.21 & 0.49 & 0.60 & 0.23 \\
Lab - Trial 2 (cm/s) & 0.29 & 0.45 & 0.20 & 0.57 & 0.74 & 0.19 \\
Lab - Trial 3 (cm/s) & 0.31 & 0.47 & 0.23 & 0.50 & 0.69 & 0.19 \\
MIT $(\mathrm{cm} / \mathrm{s})$ & 0.57 & 0.64 & 0.57 & 0.77 & 0.88 & 0.50 \\
Error & 0.90 & 0.42 & 1.71 & 0.48 & 0.29 & 1.50 \\
\hline Case & $\mathbf{1 1}$ & $\mathbf{1 2}$ & $\mathbf{1 3}$ & $\mathbf{1 5}$ & $\mathbf{1 7}$ & $\mathbf{1 9}$ \\
\hline Lab - Trial 1 $(\mathrm{cm} / \mathrm{s})$ & 0.31 & 0.56 & 0.37 & 0.63 & 0.24 & 0.36 \\
Lab - Trial 2 $(\mathrm{cm} / \mathrm{s})$ & 0.39 & 0.56 & 0.24 & 0.59 & $N / A$ & N/A \\
Lab - Trial 3 (cm/s) & 0.40 & 0.63 & 0.38 & 0.67 & N/A & N/A \\
MIT (cm/s) & 0.63 & 0.72 & 0.67 & 0.89 & 0.81 & 0.99 \\
Error & 0.75 & 0.24 & 1.03 & 0.41 & 2.38 & 1.75 \\
\hline
\end{tabular}

comparison. As with other metrics, these cases do not match the trends of other high eddy activity cases. The cases with less eddying behavior (e.g. Cases 4, 8, and 15) show a better match in plume velocity, with low errors, ranging from $29.4 \%$ to $42.2 \%$. Cases 3 and 11 have relatively high errors, which supports the results for the other metrics. The non-rotating cases (e.g. Cases 17 and 19) have the highest errors in plume velocity, ranging between $175.0 \%$ to $237.5 \%$. Whereas the shape of the plume (Fig. 2) and the plume path (Fig. 3) indicate a close match between laboratory experiment and numerical simulation, the plume area (Fig. 4) and plume velocity (Table 2) show large discrepancies.

The MITgcm's plume velocity for these cases is much higher than the laboratory experiment's plume velocity. A side view of the laboratory experiments shows that the plume descends rapidly down the slope and actually lifts off the bottom slope. The plume head develops some turbulence, which can be seen in Cases 17 and 19 in the plume images in Fig. 2. This turbulent activity is present in the MITgem images. A side view of the MITgcm plume does show the plume lift slightly, but it does not capture the eddies and turbulence from the laboratory experiments. The generation of vortical structures and the associated induced mixing present in the laboratory experiments, but absent from numerical simulations, explains the drastic difference in plume velocity for the non-rotating cases. This effect is strongest and most evident in the non-rotating cases, since the plume descends in a straight path down the slope. This explanation can be extended to all cases, since the MITgcm plume velocities are consistently larger than laboratory experiments.

\section{CONCLUSIONS}

The work presented in this paper is a comparison of laboratory experiments and MITgcm simulations run at lab-scale for density driven currents down a flat, sloping bottom. The laboratory cases are presented for varying inflow velocity, inflow density, and rotation rate of the tank, for a total of 18 different physical test cases. Results are analyzed by qualitatively comparing laboratory and numerical images, and quantitatively comparing tracer weighted plume path, plume area, and plume velocity. Cases are categorized into two groups: high eddy activity cases and low eddy activity cases. This distinction is made by the eddy activity apparent in the laboratory results. 
The cases with low eddy activity show the best match between results from the laboratory experiments and numerical simulations. In these cases, the results for plume path show that the MITgcm has more mixing and so the plume descends less downslope than in the laboratory. This result matches expectations and may be corrected at high resolutions. The resolution study shows that the numerical plume path converges to the laboratory plume path as resolution is increased. Plume area also verifies that the MITgcm matches the laboratory results well, but that the MITgcm's plume is diluted too much, likely due to numerical mixing. Lastly, the plume velocity errors for the cases with low eddy activity are lowest out of all of the cases. Overall, the MITgem matches well to laboratory results for all metrics for the low eddy behavior cases.

The cases with high eddy activity show a weaker match between results from the laboratory experiments and numerical simulations. In these cases, the plume paths match in the early stages of the overflow, but, at later stages, the MITgcm plume descends too far down the slope due to lack of mixing. This is due to a lack of resolution associated with a suppression of eddy-activity. Fewer conclusions can be made from the resolution study for the high eddy activity case. For example, it is unclear if the plume path slope will converge to the laboratory data as resolution is increased beyond $400^{3}$ points. Higher resolutions are expected to produce more mixing due to the eddying behavior, which would flatten out the slope to better match the laboratory plume path slope. However, a larger resolution study must be performed to test whether the MITgcm is able to capture the true eddying behavior. At moderate resolutions (such as $200^{3}$ used in this work), the mixing associated with eddies can be modeled by tuning the viscosity and diffusivity parameters. Thus, it is not clear that increasing resolution alone is enough. Plume area comparisons also show a poor match between the laboratory and numerical results. Lastly, plume velocity error is much higher for the high eddy activity cases.

The non-rotating cases provide insight to the overflow dynamical effect on numerical parameters in the rotating cases. The non-rotating cases show that the MITgcm is not capturing small scale eddies and turbulence, especially at the plume front. This causes the MITgcm plume to travel faster through the ambient fluid in all cases. This is a primary characteristic that is expected to improve as resolution is increased. The eddies near the plume front observed in the laboratory experiments are large and must be captured to ensure accurate simulations.

Whereas the mismatch between laboratory and numerical results is partly resolutiondependent, the effect of numerical parameters on eddying behavior and the associated overflow dynamics is vital for accurate simulations of overflows. As described in the Analysis section, the relationship between eddies and numerical parameters is a very sensitive one. With no single parameter governing the relationship, the trends change depending on the eddying regime (or spatial region). For the results presented here, the viscosity and diffusivity were chosen to be as close as possible to the real laboratory values. In general, overflow simulations are sensitive to changes in viscosity and diffusivity. Therefore, a much larger study needs to be done for a complete understanding.

\section{REFERENCES}

[1] Legg, S., Overflows and Convectively-driven Flows, Buoyancy-driven Flows, 2012, pp. 203-239.

[2] Girton, J.B. \& Sanford, T.B., Descent and modification of the overflow plume in the Denmark strait*. Journal of Physical Oceanography, 33(7), pp. 1351-1364, 2003. https://doi.org/10.1175/1520-0485(2003)033<1351:damoto >2.0.co;2 
[3] Käse, R.H., Girton, J. \& Sanford, T., Structure and variability of the Denmark Strait Overflow: Model and observations. Journal of Geophysical Research: Oceans (19782012), 108(C6), 2003. https://doi.org/10.1029/2002jc001548

[4] Mauritzen, C., Price, J., Sanford, T. \& Torres, D., Circulation and mixing in the Faroese Channels. Deep Sea Research Part I: Oceanographic Research Papers, 52(6), pp. 883-913, 2005. https://doi.org/10.1016/j.dsr.2004.11.018

[5] Foster, T.D. \& Carmack, E.C., Frontal zone mixing and Antarctic Bottom Water formation in the southern Weddell Sea. In Deep Sea Research and Oceanographic Abstracts, 23(4), pp. 301-317, 1976. https://doi.org/10.1016/0011-7471(76)90872-x

[6] Gordon, A.L., et al., Energetic plumes over the western Ross Sea continental slope. Geophysical Research Letters, 31(21), 2004. https://doi.org/10.1029/2004g1020785

[7] Muench, R., et al., A dense water outflow from the Ross Sea, Antarctica: Mixing and the contribution of tides. Journal of Marine Systems, 77(4), pp. 369-387, 2009. https:// doi.org/10.1016/j.jmarsys.2008.11.003

[8] Peters, H., et al., Mixing and entrainment in the Red Sea outflow plume. Part I: Plume structure. Journal of Physical Oceanography, 35(5), pp. 569-583, 2005. https://doi. org/10.1175/jpo2679.1

[9] Peters, H. \& Johns, W.E., Mixing and entrainment in the Red Sea outflow plume. Part II: Turbulence characteristics. Journal of Physical Oceanography, 35(5), pp. 584-600, 2005. https://doi.org/10.1175/jpo2689.1

[10] Baumert, H.Z., Simpson, J. \& Sündermann, J., Marine Turbulence: Theories, Observations, and Models. Vol. 1, Cambridge University Press, 2005.

[11] Baringer, M.O.N. \& Price, J.F., Mixing and spreading of the Mediterranean outflow. Journal of Physical Oceanography, 27(8), pp. 1654-1677, 1997. https://doi. org/10.1175/1520-0485(1997)027<1654:masotm>2.0.co;2

[12] Ilıcak, M., Özgökmen, T.M., Peters, H., Baumert, H.Z. \& Iskandarani, M., Performance of two-equation turbulence closures in three-dimensional simulations of the Red Sea overflow. Ocean Modelling, 24(3), pp. 122-139, 2008. https://doi.org/10.1016/j.ocemod.2008.06.001

[13] Legg, S., Briegleb, B., Chang, Y., Chassignet, E.P., Danabasoglu, G., Ezer, T., Gordon, A.L., Griffies, S., Hallberg, R., Jackson, L., Large, W., Özgökmen, T.M., Peters, H., Price, J., Riemenschneider, U., Wu, W., Xu, X. \& Yang, J., Improving oceanic overflow representation in climate models: the gravity current entrainment climate process team. Bulletin of the American Meteorological Society, 90(5), pp. 657-670, 2009. https://doi. org/10.1175/2008bams2667.1

[14] Snow, K., et al., Sensitivity of abyssal water masses to overflow parameterisations. Ocean Modelling, 89, pp. 84-103. https://doi.org/10.1016/j.ocemod.2015.03.004

[15] Wang, H., Legg, S.A. \& Hallberg R.W., Representations of the Nordic Seas overflows and their large scale climate impact in coupled models. Ocean Modelling, 86, pp. 76-92, 2015. https://doi.org/10.1016/j.ocemod.2014.12.005

[16] Riemenschneider, U. \& Legg, S., Regional simulations of the Faroe Bank Channel overflow in a level model. Ocean Modelling, 17(2), pp. 93-122, 2007. https://doi. org/10.1016/j.ocemod.2007.01.003

[17] Cenedese, C., Whitehead, J.A., Ascarelli, T.A. \& Ohiwa, M., A dense current flowing down a sloping bottom in a rotating fluid. Journal of Physical Oceanography, 34(1), pp. 188-203, 2004. https://doi.org/10.1175/1520-0485(2004)034<0188:adcfda>2.0.co;2

[18] Etling, D., Gelhardt, F., Schrader, U., Brennecke, F., Kühn, G., Chabert d'Hieres, G. \& Didelle, H., Experiments with density currents on a sloping bottom in a 
rotating fluid. Dynamics of Atmospheres and Oceans, 31(1-4), pp. 139-164, 2000. https://doi.org/10.1016/s0377-0265(99)00031-7

[19] Sommeria, J. \& Decamp, S., Scaling properties of dense water overflows on a continental slope. 18ème Congrès Franlccais de Mécanique (Grenoble 2007), 2007.

[20] Hallberg, R., Time integration of diapycnal diffusion and Richardson numberdependent mixing in isopycnal coordinate ocean models. Monthly Weather Review, 128(5), pp. 1402-1419, 2000. https://doi.org/10.1175/1520-0493(2000)128<1402:tiod da>2.0.co;2

[21] Davies, P.A., KÄse, R.H. \& Ahmed, I., Laboratory and numerical model studies of a negatively-buoyant jet discharged horizontally into a homogeneous rotating fluid. Geophysical \& Astrophysical Fluid Dynamics, 95(1-2), pp. 127-183, 2001. https://doi.org/10.1080/03091920108203417

[22] Song, Y. \& Haidvogel, D., A semi-implicit ocean circulation model using a generalized topography-following coordinate system. Journal of Computational Physics, 115(1), pp. 228-244, 1994. https://doi.org/10.1006/jcph.1994.1189

[23] Wobus, F., Shapiro, G.I., Maqueda, M.A.M. \& Huthnance, J.M., Numerical simulations of dense water cascading on a steep slope. Journal of Marine Research, 69(2), pp. 391-415, 2011. https://doi.org/10.1357/002224011798765268

[24] Adcroft, A., et al., Overview of the Formulation and Numerics of the MIT GCM, in Proceedings of the ECMWF Seminar Series on Numerical Methods, Recent Developments in Numerical Methods for Atmosphere and Ocean Modelling, pp. 139-149, 2004.

[25] Ravela, S., et al., A realtime observatory for laboratory simulation of planetary flows. Experiments in Fluids, 48(5), pp. 915-925, 2009. https://doi.org/10.1007/s00348-0090752-0

[26] Berntsen, J., Xing, J. \& Alendal, G., Assessment of non-hydrostatic ocean models using laboratory scale problems. Continental Shelf Research, 26(12-13), pp. 1433-1447, 2006. https://doi.org/10.1016/j.csr.2006.02.014

[27] Legg, S., Hallberg, R.W. \& Girton, J.B., Comparison of entrainment in overflows simulated by z-coordinate, isopycnal and non-hydrostatic models. Ocean Modelling, 11(1-2), pp. 69-97, 2006. https://doi.org/10.1016/j.ocemod.2004.11.006

[28] Illari, L., et al., WEATHER IN A TANK - Exploiting laboratory experiments in the teaching of meteorology, oceanography, and climate. Bulletin of the American Meteorological Society, 90(11), pp. 1619-1632, 2009. https://doi.org/10.1175/2009bams2658.1

[29] Orlanski, I., A simple boundary condition for unbounded hyperbolic flows. Journal of Computational Physics, 21(3), pp. 251-269, 1976. https://doi.org/10.1016/00219991(76)90023-1

[30] Reckinger, S.M., Petersen, M.R. \& Reckinger, S.J., A study of overflow simulations using MPAS-Ocean: Vertical grids, resolution, and viscosity. Ocean Modelling, 96, Part 2, pp. 291-313, 2015. https://doi.org/10.1016/j.ocemod.2015.09.006 